\title{
Human embryonic stem cell-derived dopaminergic neurons reverse functional deficit in Parkinsonian rats
}

\author{
Zhi-Jian Zhang ${ }^{1, *}$, Dali Yang ${ }^{1,2,{ }^{*}}$, Michael Oldenburg ${ }^{1}$, Melvin Ayala ${ }^{1}$, Su-Chun Zhang ${ }^{1}$ \\ ${ }^{1}$ Departments of Anatomy and Neurology, School of Medicine and Public Health, Waisman Center, University of Wisconsin- \\ Madison, Madison, Wisconsin, WI 53705, USA. ${ }^{2}$ Present address: Division of Cell and Gene Therapy, Department of Neurology, \\ University of Rochester Medical Center, 601 Elmwood Ave. Rochester, NY 14620, USA
}

Human embryonic stem cells (hESCs) stand for a promising cell source for obtaining functional DA neurons for cell transplantation in Parkinson's disease (PD). However, several issues such as the poor survival of grafted dopaminergic (DA) neurons, the instability of DA phenotype after transplantation and overgrowth of the graft have restricted the use of hESCs-derived DA neurons for the cell therapeutic treatment in PD. Here, we show that hESCderived dopaminergic neurons survived a long-term transplantation to the neurotoxin 6-hydroxydopamine (6-OHDA) lesioned rat striatum, and in combination with those cells newly differentiated from their progenitors, contributed to locomotive function recovery at 5 months. The animal behavioral improvement was correlated to the dopamine neurons present in the graft. Although the donor cells contained forebrain and midbrain dopamine neurons, the dopamine neurons present in the graft exhibited mainly the midbrain, or nigra phenotype, suggesting the importance of midbrain dopamine neurons in functional repair. Furthermore, progenies of grafted human cells with gradually decreased mitotic activity along the transplantation, were neurons and glia. Thus, the in vitro produced human dopamine neurons can functionally engraft in the brain in a potentially safe manner. (Supported by NIH/NINDS:U01 NS046587, R01 NS045926; The Michael J. Fox Foundation; and partly by a core grant to the Waisman Center from the National Institute of Child Health and Human Development (P30 HD03352).

Keywords: human embryonic stem cells, dopaminergic neurons, cell therapy, transplantation, Parkinson's disease, rats Cell Research (2008) 18:s37. doi: 10.1038/cr.2008.127; published online 4 August 2008

\footnotetext{
*These authors contribute equally to this work. 